\title{
Comunicación y Cultura en la Era Digital: Construir el Espacio Iberoamericano ${ }^{1}$
}

\author{
Enrique Bustamante ${ }^{2}$ \\ Universidad Complutense de Madrid
}

\begin{abstract}
Resumen: Este artículo pretende reflexionar sobre la relación entre comunicación y cultura tiendo en vista el nivel actual de integración entre ellos, especialmente en el ámbito de las industrias culturales. El paper analiza la importancia del desarrollo de políticas culturales y estrategias de cooperación iberoamericanas dirigidas al campo de la comunicación y de la cultura.
\end{abstract}

Palabras clave: comunicación; cultura; industrias culturales; políticas públicas.

\begin{abstract}
This article aims to reflect upon the relationship between communication and culture, considering their current integration especially in the context of the cultural industries. This paper analyses the importance of the development of the cultural and strategic policies of ibero-american cooperation directed to the communication and culture field.
\end{abstract}

Key words: communication, culture, cultural industry, public policies.

Resumo: Este artigo pretende refletir sobre a relação entre comunicação e cultura, tendo em vista o nível atual de integração entre estes, especialmente no âmbito das indústrias culturais. $O$ artigo analisa a importância do desenvolvimento de políticas culturais e estratégias de cooperação iberoamericanas dirigidas ao campo da comunicação e da cultura.

Palavras-Chave: comunicação, cultura, indústria cultural, políticas públicas.

\footnotetext{
${ }^{1}$ Tras la presentación original de este texto en Salvador de Bahía, en Septiembre de 2005, esta reflexión entre comunicación y cultura ha atravesado diversas etapas: presentada en México en Octubre desde la perspectiva de la esfera pública de la comunicación (Foro "México en el mundo") y en Bilbao desde el punto de vista de las industrias culturales (I Conferencia Internacional sobre Políticas Culturales.Noviembre), recuperó su significación originaria hacia la cooperación iberoamericana en el libro Iberoamérica: el mañana es hoy (A. Fraerman, Ed.2005). Aunque se trata de textos diversos y con enfoques diferentes, el presente artículo no puede dejar de ser una síntesis de esas reflexiones sobre las relaciones entre comunicación y cultura, orientadas hacia el espacio iberoamericano.

2 Enrique Bustamante es Catedrático de Comunicación Audiovisual en la Universidad Complutense de Madrid. Fundador y Coordinador de la revista Telos, es autor de numerosas obras sobre la economía del audiovisual y de las Industrias Culturales. Ha sido vocal del Consejo para la reforma de los medios públicos nombrado por el Gobierno presidido por José Luis Rodríguez Zapatero.
} 
"Necesitamos un nuevo paradigma de desarrollo en el que la cultura esté situada en el centro"

(Eduard Delgado. Sueños e identidades. 1999)

Reflexionar sobre las relaciones entre Cultura y Comunicación puede parecer, a primera vista, un ejercicio superfluo, porque ambos espacios públicos siempre han estado irremediablemente unidos. Pero los malentendidos y fracturas históricas continuas entre ambos exigen vivificar esta línea de pensamiento. En todo caso, lo nuevo de la situación actual reside en la completa integración entre comunicación y cultura y su articulación y jerarquización en las industrias culturales. Se necesita así, más que nunca un enfoque unitario e integral en las políticas públicas y de cooperación internacional en ambos campos, especialmente en las relaciones iberoamericanas.

\section{Un matrimonio indisoluble, pero incomprendido}

El matrimonio entre cultura y comunicación mantiene relaciones paradójicas y a menudo incomprendidas en nuestra sociedad contemporánea. A primera vista, sabemos que no hay cultura socialmente existente que no tenga un plan intrínseco de difusión y, por tanto, una comunicación fehaciente ante determinados sectores sociales, por pequeños que estos sean. A sensu contrario, si es difícil pensar una comunicación que no tenga aspectos creativos y deje de transmitir valores de cultura, más aun resulta inimaginable un medio de comunicación masivo que se limite a transmitir- intocable- la cultura realizada en otro lugar, aunque sólo sea porque la programación crea un nuevo sentido. Como han concluido autores que han reflexionado sobre ese tema desde hace años, "la relación entre ambos es estructural: "Una no marcha ni se explica, sin la otra" (...) si la cultura es un hecho social no hay cultura más que manifestada, transmitida y vivida por el individuo" (Caune,1995). Pero esta dependencia mutua se ha acrecentado en el seno de las Industrias Culturales, pese a sus dinámicas sectoriales diversas, en una expansión llena de sinergias, e incluso entre las artes clásicas mercantilizadas (los espectáculos en vivo, las artes plásticas, los museos y el patrimonio) cuya difusión hoy, incluso sin caer en la obsesión de las máximas audiencias, depende en buena medida de los medios de comunicación. Y sin 
embargo, la polivalencia y ambigüedad de ambos términos ha servido de palanca muchas veces para ignorar esas relaciones, especialmente cuando los intereses políticos o económicos aconsejaban su separación y aislamiento tajantes.

Los ejemplos de este divorcio entre cultura y comunicación serían interminables. Como muestra en muchos países la ubicación de las responsabilidades en cada campo en departamentos ministeriales diferentes, marcados siempre en el caso de la comunicacióninformación por el poder político directo (los Ministerios o Consejerías de Presidencia). Incluso en la historia de las actuaciones críticas aparece esta segregación, evidente en los años sesenta y setenta en los movimientos que en el llamado Tercer Mundo, pero sobre todo en América Latina, plantearon la reivindicación de un reequilibrio de los flujos de información que dio origen al deseado NOMIC (Nuevo orden de la Información y la Comunicación), con un sesgo general-aun con excepciones sobre el cine o la música- por la información de actualidad (con los medios o las agencias regiones o nacionales por ejemplo como solución). Y sin embargo, el documento culminante de aquel movimiento, el Informe MacBride, hoy celebrado a los 25 años de su edición, proclamaba claramente "la interdependencia de la cultura y la comunicación" y situaba a esta última como "vector fundamental de la cultura", y a los medios masivos como "medio principal de acceso a la cultura y a todas las formas de expresión creada", que crean además "una nueva cultura”.(MacBride, 1980).

Desde entonces, las interrelaciones entre cultura y comunicación masivas, no han cesado de crecer y estrecharse por la vía de la hegemonía alcanzada por las Industrias Culturales, y de la publicidad y los medios de comunicación en su seno. Se ha podido escribir así recientemente que "en este doble movimiento de la cultura a la comunicación y de la comunicación a la cultura y la sociedad se mueve gran parte del pensamiento contemporáneo. De ahí que hoy nos resulte tan inconsistente, tan artificial, pensar la cultura y la comunicación como una oposición disyuntiva más. Sin embargo, esta tentación todavía subsiste en buena parte de las políticas culturales y aun de estudios humanísticos" (García Canclini, 1999). La misma historia de la Unión Europea, la de más larga experiencia en un proceso de integración regional de países que se conoce, muestra una larga trayectoria de políticas culturales iniciadas en el campo audiovisual que se aíslan 
cuidadosamente de las políticas de comunicación- presuntas integrantes de la soberanía política nacional- para no tocarse más allá de algunos puentes imperfectos y mal instrumentados, como la Directiva de TV sin Fronteras.

Hoy, cuando se produce el "regreso de la política cultural y comunicativa" (Zallo, 2005), después de años de ausencia de debate, y cuando los sistemas culturales y comunicativos son puestos en cuestión en muchos países, la vigilancia sobre estas articulaciones mutuas son más importantes que nunca. Porque en medio de la integración tecnológica (la convergencia digital especialmente) y de la concentración e integración económica de las Industrias Culturales en grandes conglomerados transnacionales y nacionales, una respuesta separada sería un auténtico desastre para su eficacia y su mantenimiento. En sentido positivo, se juega además el fin de unas políticas de Estado que se han dedicado no pocas veces a cultivar el pasado (el patrimonio) o las artes más elitistas, dejando abandonado el futuro (las industrias culturales, incluyendo los medios de comunicación) al mercado. O que, incluso en esos casos, han centrado su protección sobre la producción de bienes culturales, olvidándose de crear condiciones propicias para la creatividad- en el monte de la cadena de producción- y de fomentar la distribución y la promoción (en el valle) indispensables.

Las luchas de los últimos años, centradas primero sobre la excepción cultural y luego sobre la diversidad, están contribuyendo paulatinamente a superar esas viejas divisiones. Porque la diversidad, entendida correctamente como posibilidad real de elección del usuario, exige una actuación de futuro en ambos campos simultáneamente, y centra por tanto las políticas públicas en el ciudadano-consumidor de donde nunca debieron desviarse.

Por una parte, el debate actual, reforzado en muchos países, sobre el papel de los medios y el servicio público, y sobre la necesidad de reguladores independientes capaces de armonizar el sistema de comunicación en su conjunto al servicio del interés general, muestra que una parte importante de la sociedad cuestiona el deterioro progresivo de la esfera pública. Así, coincidentemente en casi todos los países de la Unión Europea y en buena parte de Latinoamérica, el cuestionamiento del sistema de medios masivos de 
comunicación y el papel que en su seno juega la ciudadanía están a la orden del día. Y este debate no viene sólo ni principalmente de la investigación o de la academia sino de las asociaciones ciudadanas de todo tipo y de los partidos políticos e incluso gobiernos que se hacen eco de la extensión de esas reivindicaciones. Se centra especialmente en el sector audiovisual y, muy específicamente, en la televisión, pero abarca también a los otros medios de comunicación y se expande cada vez más hacia Internet y las nuevas redes digitales. Trae además, como novedad significativa respecto a los debates del pasado, una orientación más amplia, que trasciende con mucho las fronteras del pluralismo político para situarse claramente en el campo de la cultura, de la creación y transmisión social de los contenidos simbólicos de todo tipo.

La discusión sobre los medios masivos se integra así, más que nunca, en la nueva fuerza creciente que la cultura está alcanzando hoy en la agenda internacional como base ineludible del desarrollo integral, y la diversidad en tanto condición sine qua non de esa cultura. Como afirmaba el pionero Eduard Delgado, "Los medios, el patrimonio y las políticas culturales deben ser considerados de manera integrada y están íntimamente ligados a las políticas educativas". (Delgado, 1999). De forma que es imposible separar y aislar este fenómeno del movimiento civil, de amplitud creciente, en torno a la creación de observatorios críticos de las Industrias Culturales, ode la creación en muchos países de "coaliciones por la diversidad" que reúnen a asociaciones de autores, a entidades culturales y ciudadanas e incluso a organismos estatales, en torno a la batalla por la aprobación y aplicación del Convenio por la Diversidad auspiciado por la UNESCO.

Ello no quiere decir que se hayan superado de golpe las fracturas conceptuales y políticas heredadas, sea por las hipotecas conceptuales del pasado o por políticas coyunturales mal entendidas. Es curioso así, que con la cobertura de la UNESCO, sus anuarios sobre cultura y sobre comunicación se hayan alternado en los últimos años, pese a una evidente temática común y a los vasos comunicantes de sus problemáticas. Y de la misma forma, la justamente celebrada Convención por la diversidad, aprobada por la Conferencia General de Octubre pasado, pese a la frontal oposición de los Estados Unidos e Israel, ha sido criticada por minimizar la diversidad de los medios de comunicación, -y silenciar totalmente los peligros de su concentración-, citada sólo de soslayo en dos 
párrafos, el que afirma (punto 12 del preámbulo) que "la libertad de pensamiento, de expresión y de información, así como la diversidad de los medios de comunicación permiten el florecimiento de las expresiones culturales en el seno de las sociedades"; y el que, recopilando las acciones posibles, habla de medidas que "apuntan a promover la diversidad de los medios de comunicación, incluso a través del servicio público de comunicación". No es extraño pues que un avezado analista, recordara a este respecto que "la construcción de políticas culturales es difícil de concebir sin hacer una incursión en las políticas de comunicación" (Mattelart, 2005), pero tampoco que planeara en torno a la UNESCO el fantasma de la autocensura en recuerdo de la feroz ofensiva de los grupos mediáticos occidentales contra el NOMIC en los años 70 y 80, culminada en el Congreso de Talloires.

Y sin embargo, en la medida en que los Estados-nación están siendo superados también por abajo en sus competencias, antaño exclusivas, sobre estos capítulos, se pueden reseñar documentos mas valientes en este punto. Como la propia Agenda 21 de la Cultura, que en su punto 30 se compromete a "potenciar el papel estratégico de las industrias culturales y los medios de comunicación locales, por su contribución a la identidad local, la continuidad creativa y la creación de empleo", para pronunciarse todavía más claramente en el punto 32 por "implementar políticas que tengan como objetivo la apertura de medios de comunicación públicos en el ámbito local, así como su desarrollo de acuerdo con los intereses de la comunidad, siguiendo los principios de pluralidad, transparencia y responsabilidad", y por suscitar la lucha legal contra la concentración (punto 53).

\section{Las nuevas amenazas de oligopolio:}

La fuerza internacional que está recuperando ese interrogante social sobre el sistema hegemónico de comunicación y cultura, en medio de un discurso mediático y político abrumador a favor del todo mercado, evidencia una reacción fundamentada sobre las vivencias cotidianas frente a las transformaciones que recorren en las dos últimas décadas el mundo de la comunicación y la cultura. Y frente a las tendencias hegemónicas que hoy se perfilan en este terreno, especialmente en el desarrollo de las redes digitales y 
del modelo de sus servicios de información (redes y servicios de todo pago, desarrollo empresarial o individual en el plano doméstico).

De un lado, comenzando por el mundo analógico, la creciente concentración está suscitando nuevas preocupaciones que van mucho más allá de las tradicionales inquietudes sobre su amenaza al pluralismo del espacio político; y nuevas investigaciones cuantitativas y cualitativas, ante la ineficacia de los indicadores convencionales al uso, que abarcan la creciente integración vertical, -desde la producción hasta la distribución y la difusión-, así como los procesos de diversificación multimedia y sus consecuencias sobre los procesos de creación y sobre la libertad de elección del usuario. Se cuestiona así, de paso, las políticas de desregulación general de dos décadas y el desarme de muchos estados de su regulación anti-trust, realizada en paralelo a la glorificación de los "campeones nacionales", los grupos privados más fuertes de cada país, teóricamente destinados a defender simultáneamente nuestra economía y nuestra cultura, rodeados de una filosofía ad hoc (la fortaleza como supuesta garantía de independencia, la nacionalidad de la casa madre como promesa de renacionalización de la creación).

Pero la investigación y los interrogantes sociales van hoy mucho más lejos, y comienzan a cuestionar a los grupos multimedia globales y locales, en su función capital de productores y distribuidores de contenidos simbólicos (culturales e informativos). Se empieza a estudiar así cómo la carrera hacia el gigantismo global y multimedia de algunos grupos globales (estadounidenses, pero también europeos y japoneses) ha impreso una perentoria exigencia de beneficios máximos y crecientes, por la financiarización de las corporaciones (apelación creciente al mercado de capitales) y la necesidad de expansión permanente (la talla máxima como tamaño óptimo), que sólo por la aplicación masiva del marketing a la distribución e incluso a la creación puede intentar mantenerse. Y se inician estudios empíricos destinados a verificar el efecto de esas estrategias sobre las culturas locales, sobre los creadores y las PYMES, sobre los elementos básicos de la diversidad nacional e internacional (VV.AA., 2005).

Según estos trabajos, la amenaza de imposición de una cultura MacDonald por la globalización , tema de discusión general en los últimos años, yerra el camino y desvía el 
debate público. Y no sólo porque la homogeneización inherente a la distribución mundial de los productos globales aparezca perfectamente compatible con el mantenimiento e incluso la revitalización de la producción y los mercados domésticos. Sino porque las grandes corporaciones multinacionales tienden a ocupar simultáneamente los mercados masivos y los nichos locales ricos, controlando ambos por su poder sobre las redes de distribución, sin que su dominio lleve necesariamente aparejada una rarefacción de los productos y servicios culturales, (en ocasiones se impulsa por el contrario una saturación de oferta, para mejor asfixiar a los competidores). Se trata en suma de una "diversidad de mercado", con la imposición del todo mercancía y de la máxima rentabilidad sobre las especificidades y dinámicas culturales, con tendencia a asfixiar la ecología delicada en que radicaba la diversidad (creadores-productores-distribuidores independientes, PYMES como cantera de innovación, espacios de no-mercado como resortes de equilibrio...).

Todo ello, apunta al dominio creciente de una reprocultura (Achille, 1997), de una cultura clónica (Bustamante, 2003), basada en la repetición incesante de éxitos comprobados, conservadores en la estética y descocados en ocasiones en la ética social, que tiende a marginalizar o ahogar toda creación innovadora, vanguardista, revulsiva, o localmente genuina en beneficio de la búsqueda incesante de mercados masivos .

En concreto, los estudios realizados permiten aseverar que las estrategias "pull" de los grandes grupos (en el disco, el cine o el libro...), aprovechan la aceleración de las ventas en todas las ventanas o mercados, mediando campañas intensivas de comunicación promocional, para dominar los mercados masivos e incluso los nichos minoritarios pero ricos. Construyen así círculos virtuosos de mercado, con prácticas anticompetitivas y opacas, en los que a más promoción se consiguen más ventas que a su vez dan lugar a más promociones hasta cifras de decenas o centenares de miles de productos vendidos en poco tiempo. Por el contrario, y como consecuencia de esas dinámicas, las PYMES o las creaciones minoritarias, a falta de espacios de comunicación que les permitan salir de circuitos marginales, a duras penas consiguen amortizar sus costes, viéndose sometidas a altas tasas de mortandad. Un proceso complejo, que se caracteriza por anclarse, como resorte indispensable, sobre los dispositivos de comunicación en donde se centra esa "economía de embudo", en la que los agentes más débiles corren todos los riesgos de la 
innovación, con escasas posibilidades de capitalización y consolidación, mientras el oligopolio cómodo se instala en los grandes grupos integrados desde la creación hasta la distribución.

Lejos de toda idea simplista de la cultura "MacDonald", o de visiones maniqueas de "invasión exterior", las preocupaciones incluyen también a los grandes grupos nacionales o regionales de la periferia. Así, en una investigación recientemente publicada en sus resultados provisionales sobre las "majors iberoamericanas" en la cultura y la comunicación (desde Televisa, Globo o Venevisión hasta Planeta, Telefónica o Prisa, pasando por grupos "medianos" de orden regional como Abril, Azteca, Vocento, Clarín...) observábamos una trayectoria similar: desde los grupos familiares de origen a la implantación general del management moderno, desde los reforzamientos nacionales hasta la expansión regional y multimedia con el recurso a la bolsa o los capitales externos, y con un tejido cada vez más intrincado de alianzas entre grupos globales y regionales. Se constataba cómo se realizaba en paralelo, aunque hagan falta muchas más investigaciones empíricas, un proceso de cambio radical de sus modos de producir y valorizar las creaciones culturales y comunicativas cuya doble cara, de globalización económica y simbólica, quedaba señalada en el crisol simbólico de Miami y su papel en la cultura "hispana" (Bustamante, Miguel, 2005).

Estos estudios adelantan asimismo procesos prolongados en las redes y los soportes digitales. En primer lugar, porque en las últimas décadas, en las telecomunicaciones también se ha vivido una generalizada privatización de las redes, con dinámicas empresariales de beneficio máximo, y con repercusión de costes a todas las tarifas, incluyendo los operadores de la difusión punto-masa en las ondas hertzianas, el cable o el satélite.

En segundo lugar, y en lo que respecta a los contenidos, la desmaterialización digital de la cultura y la información está implicando un cambio radical de su naturaleza económica, asimilada a la de la radio-televisión (costes marginales por espectador iguales a cero), obviando el coste de conexión. Pero esa virtud, que significaría en teoría grandes ventajas de ahorros de costes para el usuario y de mejores remuneraciones para una más 
extensa base de creadores, encierra también economías de escala muy fuertes que impulsan a la concentración. De forma que todas las promesas de diversidad encerradas en esos cambios, están puestas en cuestión ahora por un movimiento de concentración que, aunando globalización del mercado y posibilidades de las nuevas redes, ha puesto en marcha movimientos múltiples, desde la integración vertical entre redes y gigantescas carteras de contenidos hasta la fusión entre operadores de telecomunicaciones y fabricantes de software con grandes corporaciones mediáticas, o alianzas que refuerzan aun más el poder oligopolista por sectores y, especialmente, en forma transversal multimedia,

En definitiva, junto a las grandes oportunidades abiertas por la Era Digital a la cultura, a la comunicación y la formación, lo que verificamos hoy de forma dominante es, junto a fracturas digitales múltiples, un riesgo inédito de concentración (al mismo tiempo contra el pluralismo y contra la competencia) en grandes grupos que comienzan a colonizar completamente los nuevos dominios (Low, 2001). Así, en el mundo digital como en el analógico, conglomerados de peso desproporcionado, que ante el retroceso del Estado, "disfrutan de un amplio margen de maniobra" (...) lejos de favorecer el pluralismo y los ideales democráticos, refuerzan el orden ya jerarquizado al integrar a todos dentro de un mismo sistema coercitivo" (Ortiz, 2005). Y ello en paralelo a un salto cualitativo en la comercialización (commodification) de la cultura y a un "impulso sin precedentes a la internacionalización de los flujos de los productos culturales" (Miège, 2000) que provocan unidos una estratificación mercantil por encima de los territorios y las fronteras (Schiller, 1997; García Canclini, 1999).

De esta forma, la problemática del desarrollo de las redes digitales ha complejizado aun la problemática de las políticas y estrategias de comunicación y cultura, -y sus parentescos y separaciones de paso con las políticas educativas-, al introducir una nueva variable, especialmente desde los años noventa, en forma de políticas de Sociedad de la Información. Una nueva línea de actuación de los Estados, de los organismos de integración regional e incluso de las regiones y municipios, cuyos lazos evidentes e imprescindibles con las actuaciones en cultura y comunicación han caído de nuevo en la ambigüedad ideológica y práctica. 
Como evidencian en este terreno las actuaciones de la Unión Europea desde 1994 hasta la actualidad (desde el Informe Bangemann a la cumbre de Lisboa), movilizadas inicialmente al calor del Proyecto Al Gore, las medidas de Sociedad de la Información han sido situadas con frecuencia en los espacios de la política económica e industrial, y centradas en su esfuerzo sobre las redes y los equipos, con olvido de los contenidos y servicios cuya importancia estratégica sin embargo se repetía con frecuencia. Traspuestas en ocasiones al ámbito directo de la comunicación y la cultura (como en el Libro Verde de Programas estratégicos de 1994, o en recientes planes de apoyo al multimedia), la mayor parte de estos planes olvidaba las dinámicas de la creatividad y del consumo cultural de la sociedad para adoptar una visión economicista y vertical de oferta-demanda que imaginaba que la tecnología se imponía por sí sola, acompañada en el mejor de los casos por unos servicios "intangibles" o "de contenido", conceptos difusos nacidos en los operadores de redes que permiten evacuar los lazos molestos con la cultura y la comunicación social. En consonancia con esta línea, muchos países europeos han confiado el destino de la "Sociedad de la Información para todos" (un eslogan que retoma retóricamente el modelo social europeo) a un proyecto meramente industrial, encargado en exclusiva a los grandes grupos de redes y programas informáticos.

En contraste fuerte con estas actuaciones, la llegada de las tecnologías digitales y de las infraestructuras basadas en ellas han puesto a la comunicación y la información en el centro del sistema y a su regulación y modelo como axial del destino de la sociedad entera. El propio consenso social sobre la importancia nodal de las nuevas tecnologías de la comunicación revela que la formación y la información, la cultura en una palabra, están en la base no ya solo del pluralismo sino de todo desarrollo económico y social posible para los individuos, las regiones y los pueblos, (Delcourt, 2001). Porque, como se ha dicho con aplastante lógica,"no se puede a la vez mantener el discurso de un papel crucial de Internet en la sociedad futura (...) y contemplar una regulación a minima bajo control de los operadores económicos de Internet. Es decir, Microsoft, AT \& T, Bertelsmann, Vivendi, Murdoch, etc” (M.Dagnaud/M. Bonnet, 2000). Más directamente aun, se ha señalado que "se hace necesario que los Estados asuman que la Información y la Comunicación configuran un sector de los servicios públicos tan estratégico socialmente como los 
servicios de salud y educación, y así debería empezar a aparecer en los documentos de política nacional con nombre propio, el de Servicio Público de Información y Comunicación” (Martín Barbero. 2005)

¿Cuáles deberían ser las líneas generales de esa actuación estatal y civil a un tiempo?. Del análisis de la historia y transformaciones de las políticas culturales y de comunicación, y de sus experiencias internacionales más cualificadas, hemos apuntado trazos maestros (Bustamante, 2003), que aquí sólo podemos sintetizar:

1.-En el terreno de las redes de comunicación, es preciso potenciar la expansión de las redes digitales menos costosas y más proclives al servicio universal, en el sentido de asequible pero también asequible a todos los ciudadanos, entre las que el DAB y la TDT descuellan ampliamente, pero también otras tecnologías como las radiofrecuencias y wireles en general. Más allá de la tecnología, es el modelo mismo de acceso el que debe ser reconsiderado: frente al acceso individual y de pago según el patrón estadounidense, el gratuito y colectivo que cuenta ya con múltiples experiencias nacionales. Lo que conlleva un concepto de servicio colectivo universal, de "geometría variable", adaptable a la banda ancha y a la evolución tecnológica de las posibilidades de comunicación a distancia.

2.-Pese a todas las frustraciones del pasado, es preciso seguir reivindicando una política preventiva anti-trust capaz de poner a coto a la concentración, por encima de límites peligrosos en cada sector e hilera productiva y en cada mercado de referencia relevante (nacional, regional, local...), además de en la concentración cruzada o multimedia, en las industrias culturales y con las redes y soportes digitales.

3.-Más importante aún en términos positivos, es necesario recrear una política sistemática de apoyo a la creación, la edición y la difusión de la cultura por parte de las Pymes, de las instancias asociativas, del "tercer sector" (ni Estado ni gran "mercado"), de la sociedad civil en una palabra tanto en términos analógicos como cara al entorno digital de futuro. Y, aunque ciertamente las nuevas redes invalidan muchas medidas actuales, como las cuotas, no faltan en la experiencia reciente herramientas útiles para esa labor y adecuadas a los nuevos marcos: desde la reserva de capacidad en las diversas 
infraestructuras para los productores o programadores independientes (open acces), o las exigencias de transporte de la señal de los canales públicos (must carry), hasta la obligatoria inversión de los difusores en esa producción independiente o la discriminación positiva en las subvenciones y el tratamiento fiscal hacia las Pymes (por ejemplo para la digitalización de sus librerías y su nueva comercialización). El objetivo de las políticas culturales y de comunicación, con la diversidad como meta, es en definitiva, "un peso equitativo de los tres modelos (...) que salvaguarde el pluralismo en cada modelo"( Yúdice, 2002): el equilibrio en el mercado (entre profesionales, Pymes y grandes empresas), en el Estado (con gestión y control autónomo de sus funciones) y con el tercer sector (asociativo y no lucrativo).

4.-Restaurar el objetivo central de las políticas culturales y de comunicación, el ciudadano, en el pasado muchas veces desviado hacia políticas de oferta simple (productores, editores), exige un esfuerzo acrecentado en la educación y en la comunicación cultural, tanto respecto a los usos tradicionales como en relación a las nuevas prácticas culturales en nuevos soportes.

5.-Un factor más insustituible que nunca reside en medios públicos y ciudadanos, reforzados y articulados, a todas las escalas (estatal, pero también regional o local) sobre todo con base en las posiciones mantenidas en la radio y la televisión. Pero la expansión de este espacio de no-mercado a las nuevas redes (portales y buscadores en Internet, bibliotecas y registros públicos en red por ejemplo) es una exigencia natural de la Sociedad de la Información, que requiere asimismo pensar la comunicación y la cultura de forma activa por los usuarios.

\section{Por un espacio cultural y comunicativo ibero/ euroamericano}

Las políticas públicas de muchos Estados han pecado durante años de las miopías señaladas anteriormente en un sentido múltiple: aislamiento radical entre la cultura (reducida muchas veces a la cultura clásica o a alguna industria cultural aislada como el cine) y las estrategias comunicativas, y de ambas respecto a los programas de Sociedad de la Información; visiones proteccionistas nacionales a corto plazo que impulsaban a su vez estrategias autárquicas y cortocircuitaban por tanto la construcción paulatina de espacios y 
mercados regionales e internacionales potentes. Y todo ello en medio de la permanencia reiterada de un discurso retórico sobre la cooperación internacional como intercambio de culturas, que resultaba desmentido por los hechos, tanto en el plano de la diversidad cultural como en el de la propia escala del tejido industrial. Las políticas culturales de la Unión Europea, durante dos décadas, constituyen en ese sentido un paradigma extremadamente interesante para analizar cómo las ricas experiencias logradas han quedado lastradas por la falta de voluntad financiera, pero también y muy especialmente por su fragmentación de la realidad en ámbitos inseparables y, finalmente, por los egoísmos nacionales que primero, frustraban el objetivo de construir un espacio común y, luego, aislaban a la cultura europea de la creatividad de países y regiones terceros.

Evidentemente, no se trata de poner en cuestión la indiscutible legitimidad de los Estados, y de las propias sociedades nacionales o locales, para defender y proteger su producción cultural, justamente reforzada ahora por un instrumento jurídico internacional. Sino de cuestionar de raíz una visión proteccionista nacional que, si se ha mostrado insuficiente y miope a escala del mundo analógico, amenaza degenerar en desastre en un mundo digital caracterizado por la multiplicidad de las ofertas y la hiperfragmentación de la demanda y de los usos; $\mathrm{Y}$ de interrogarse a fondo sobre los resultados en ambos escenarios de una empecinada política que confronta la cultura con la economía, y asimila lo público al pasado o, al menos a un elemento subsidiario y subordinado al mercado.

La política audiovisual y cultural en general de España hacia América Latina, y la de los países iberoamericanos entre sí, no ha estado exenta de errores similares a los que antes mencionábamos. Durante años primó la retórica de "espacios" comunes identitarios sobre la lengua y las sensibilidades culturales, mientras cada país sólo contemplaba a los otros como potenciales mercados de expansión comercial. Luego, se fueron abriendo caminos de cooperación e intercambio pero preferentemente ceñidos a campos "legitimados" de cultura clásica, mientras en las industrias culturales y los medios de comunicación primaba la complacencia por los logros expansivos de los grandes grupos en el terreno regional o internacional. De forma subyacente, se presuponía que el mercado, y nuestros "campeones nacionales" iban a ir construyendo ese espacio común de futuro. Aunque los datos empíricos revelaran que, más allá de terrenos de monocultivo como la telenovela o algún 
nicho musical, el progreso en la construcción de un espacio común en las industrias culturales de masas brillaba por su reiterada ausencia.

Una iniciativa pareció romper esta dinámica en los últimos años, el programa Ibermedia, que ha mostrado ricas experiencias, pese a sus magros presupuestos, que mostraban la viabilidad de la construcción progresiva de un espacio común audiovisual a medio plazo. Sin embargo, este programa primó también a la producción frente a la distribución efectiva o la promoción; y olvidó generalmente la concepción de un audiovisual integral, especialmente de la imprescindible pata televisiva, a falta de la cual resulta imposible completar la financiación y amortización de los productos, o cambiar la imagen de los públicos iberoamericanos sobre su propia identidad audiovisual.

Los cambios políticos en muchos países del área han comenzado sin embargo a mostrar una nueva sensibilidad. Por ejemplo, al filo de la cumbre iberoamericana de Salamanca, comenzó a barajarse la necesidad de la creación de canales televisivos comunes que conectaran y articularan una cooperación sistemática en este campo y, a través suyo, del conjunto de la industria cultural. Aunque finalmente, el asunto se aplazó por falta de estudios previos que determinaran las demandas concretas que debían orientar el proyecto.

Sin ánimo exhaustivo, podemos así apuntar proyectos y líneas de acción que podrían impulsarse en el futuro próximo en esa perspectiva integral que venimos defendiendo:

-La experiencia de Ibermedia podría ampliarse a campos como el de la industria musical y discográfica, o al de las Pymes de la industria editorial de libros, o al de las revistas culturales de todo tipo, en donde ha habido algunas experiencias exitosas de cooperación.

-La condición sine qua non del éxito de esas empresas, sería la aportación de un serio apoyo comunicativo, especialmente desde la radio y la televisión pública y comunitaria, en donde RTVE y alguna otra televisión pública fuerte, como TVN de Chile, podían actuar como locomotora inicial de estos proyectos, en estrecha alianza con otras entidades regionales y locales, como las televisiones autonómicas públicas españolas, como promotoras de una alianza permanente con las restantes televisiones públicas 
iberoamericanas, en un foro institucionalizado como el que las televisiones francófonas han conseguido desde hace años.

-Las nuevas redes digitales, como la TDT o el satélite permiten hoy encontrar instrumentos valiosos al servicio de esta tarea, como la creación de canales cooperativos para todo el área, un viejo sueño, alentado por muchos pensadores latinoamericanos en los últimos años (como Octavio Getino, Martín Barbero o García Canclini), y que resultó ya frustrado cuando el canal Hispavisión, también decidido en una cumbre iberoamericana, se desvió de sus objetivos iniciales quedando en un canal de documentales con escasa cooperación y nula orientación regional.

En el "Informe para la Reforma de los Medios de titularidad del Estado" entregado al Gobierno español en Febrero de 2005, proponíamos por ello dos canales diferenciados, digitales terrestres y abiertos en España, por satélite, cable o digital terrestre según las circunstancias de cada país en Latinoamérica. En primer lugar, un canal educativo-cultural, que debía aprovechar las reservas y experiencias de la actual ATEI, con una programación sistemática durante parte del día, ofreciendo en el resto de las emisiones una ventana abierta a todos los ámbitos de creatividad cultural iberoamericana, clásica y moderna, así como una cooperación con la cultura europea que una participación más intensiva en ARTE podría abonar inicialmente. De otro lado, una reforma profunda de Hispavisión, que proponíamos llamar Ibervisión (por la imprescindible integración con Portugal y Brasil), abierta a todos los géneros del audiovisual, pero especialmente al largometraje, la ficción televisiva y los documentales, como genuino producto de nuestro audiovisual común, en estrecha integración con el programa Ibermedia, capaz no sólo de aportar una nueva vía financiera a este sino también de "construir una nueva imagen pública de la especifidad de nuestra creación audiovisual”.

-Con similares finalidades, en torno a las emisoras públicas o las redes asociativas, podrían crearse portales y buscadores que ayudaran a acceder a los contenidos culturales y comunicativos iberoamericanos. Un trabajo paralelo sería acentuar la cooperación entre PYMES de la producción y la distribución en cada sector, de cara a la información y venta, 
off line y on line, en donde ya existen experiencias nacionales avanzadas, con posibles ayudas para la digitalización de los archivos y su comercialización.

Tal tipo de programas no necesariamente debería ser impulsado o instrumentalizado por los Estados centrales, sino también por los movimientos asociativos, por las entidades gestoras del derecho de autor que ya están formando redes regionales, por el tercer sector no lucrativo. Una cooperación horizontal que debería privilegiar el papel de los pequeños países del área para compensar sus limitaciones, y potenciar la función de las pequeñas y medianas empresas audiovisuales y sus redes de cooperación trasatlántica, y no sólo la intervención de los grandes grupos multimedia de este ámbito.

En paralelo a la consolidación de estos avances, debería intensificarse la cooperación euro-iberoamericana, y de la U.E. con los procesos de integración regional como el MERCOSUR, en donde la conciencia sobre la cultura va también ganando terreno. Los instrumentos para estas nuevas políticas públicas no faltan, especialmente para la cooperación entre Europa y América Latina. Las cumbres mixtas (Rio, 1999; Madrid, 2002; Guadalajara, 2004) y los acuerdos económicos suscritos entre ambas regiones son el marco idóneo para esa tarea, aunque hasta ahora no hayan contemplado con relieve ni al audiovisual ni a la cultura.

La apertura de estos nuevos caminos, sobre la base de los recursos existentes, permitiría dar un salto cualitativo hacia la construcción de ese espacio iberoamericano de cultura tantas veces evocado. Sobre la convicción, insoslayable en la globalización, de que sólo juntos podemos salvar nuestras identidades y nuestras industrias culturales. 


\section{Referencias bibliográficas:}

Achille, Y., 1997. "Marchandisation des Industries Culturelles et developpement d'une reproculture". Sciences de la Société, nº 40. Toulouse.

Bustamante, E. (coord..), 2003.Hacia un nuevo sistema mundial de comunicación. Las industrias culturales en la era digital. Gedisa. Barcelona.

Bustamante, E./ Miguel, J.C. 2005. "Les groupes de communications ibéroamericains à $\mathrm{l}^{\prime}$ heure de la convergence". Réseaux, $\mathrm{n}^{0} 131$. París.

Caune, J.,1995. Culture et Communication. Convergences théoriques et lieux de médiation. Presses Universitaires de Grenoble. Grenoble.

Dagnaud, M./ Bonnet, M. 200o. Médias: promovoir la diversité culturelle. Comisariat Géneral du Plan. Paris.

J. Delcourt., 2001. "Les Convergences entre Economie et Culture". En Peten y otros, 2001.

Delgado, E. (Dir.), 1999. Sueños e identidades. Interarts/ Península.Barcelona.

Garcia Canclini, N. 1999. "Opciones de políticas culturales en el marco de la globalización”. En Informe Mundial sobre la Cultura. Unesco/Acento editorial. Madrid.

Martín Barbero, J. 2005. "Políticas de interculturalidad". Observatorio nº 2. Dossier Diversidad Cultural. Abril. Buenos Aires.

Martín Barbero, J. “Cultura y Medios de Comunicación”. Ver VV.AA., 2005.

Mattelart, A., 2001. "Vers une globalisation?”. Réseaux, nº 100. CNET/Hermes. Paris.

Mattelart,A. 2005. "Diversidad cultural. Batalla en la UNESCO”. Le Monde Diplomatique, ed. España. Octubre.

MacBride, S. (coord..)1980. Un solo mundo, voces múltiples. UNESCO/FCE. México.

Miège,B., 2000. "Producción cultural y pluralismo cultural". En Informe mundial sobre la comunicación y la Información. 1999-200o. Unesco. Cidoc. Madrid.

Miège. B. (coord..), 2005.Dossier "La concentration dans les industries du contenu". Réseaux, $\mathrm{n}^{\circ}$ 131. París.Ed. Hermes.

Ortiz, 2005. "El contexto mundial y el iberoamericano". Ver VV.AA., 2005.

Schiller,D., 1997. "Internet, terrain de jeu pour les publicitaires". Le Monde Diplomatique. Noviembre. Paris.

Yúdice, G., 2002. "Industrias culturales, diversidad cultural y desarrollo iberoamericano". En García Canclini, N.(coord..) Iberoamérica, 2002. Diagnóstico y propuestas para el desarrollo cultural.OEI/Santillana.México.

VV.AA., 2005. Cultura y sustentabilidad en Iberoamérica. Interarts/OEI. Madrid.

Zallo, R.2005. "El regreso de la política cultural y comunicativa". Telos, no 64. JulioSeptiembre. 2005. 